\title{
Notes on Early Land Plants Today. 24. What is Protolophozia? (Cephaloziellaceae, Marchantiophyta)
}

\author{
JIŘÍ VÁŇA ${ }^{1}$, LARS SÖDERSTRÖM ${ }^{2,4}$, ANDERS HAGBORG ${ }^{3}$ \& MATT VON KONRAT $^{3}$ \\ ${ }^{\prime}$ Department of Botany, Charles University, Benátská 2, CZ-12801 Praha 2, Czech Republic; vana@natur.cuni.cz. \\ ${ }^{2}$ Department of Biology, Norwegian University of Science and Technology, N-7491 Trondheim, Norway; lars.soderstrom@bio.ntnu.no \\ ${ }^{3}$ Department of Botany, The Field Museum, 1400 South Lake Shore Drive, Chicago, IL 60605-2496, USA; hagborg@pobox.com, \\ mvonkonrat@fieldmuseum.org \\ ${ }^{4}$ Author for correspondence
}

Protolophozia (Schuster 1968: 474) Schljakov (1979: 204) (三Lophozia subg. Protolophozia R.M.Schust.) is a segregate genus of Lophozia (Dumortier 1831: 53) Dumortier (1935: 17). It is mainly subantarctic and includes mostly rare species with narrow distribution ranges. Most of the species are not well known. The New Zealand species were described and discussed by Schuster (1968) and recently also by Engel \& Glenny (2008) with overviews of known taxa by Schuster \& Engel (1975) and Schuster (2002).

To date, 17 species have been referred to Protolophozia or Lophozia subgen. Protolophozia. Among them is Lophozia (Protolophozia) ciliata Söderström et al. (2000: 3) a Lophozia s.str. (De Roo et al. 2007). Protolophozia debiliformis (Schuster \& Damsholt 1987: 326) Konstantinova \& Czernjadieva (1995: 112) is regarded a synonym of Barbilophozia sudetica (Hübener 1834: 142) Söderström et al. (2010: 50) or a sister species to it (Vilnet et al. 2010). The only other species formally transferred is Protolophozia elongata (Stephani 1902: 41) Schljakov (1979: 204). These three are the only species studied molecularly confirming the position of the first two. Protolophozia elongata was neither grouped with Lophoziaceae, nor with Anastrophyllaceae (Vilnet et al. 2011). Because comparative molecular data is not available for any of the of the Southern Hemisphere taxa, their relationship to Protolophozia elongata is obscure. We are sceptical about including it in Protolophozia, but leave it there until more data is available.

The genus is most probably heterogeneous and familial placement is uncertain, but species taxonomy is also uncertain. The species delimitations are sometimes based on characters that may be plastic and should be further investigated, but this is currently impossible due to lack of material. Schuster \& Engel (1975) recognized three sections that may or may not be natural groups. In addition, the Lophozia androgyna / autoica / nivicola / subalpina / tasmanica group from New Zealand and Tasmania forms a complex (Schuster 2002), and some taxa may not deserve recognition, at least not at species level.

Even if many of the species are poorly known, Lophozia lancistipa is perfectly described and illustrated by Grolle (1971) and, moreover, illustrated and discussed by Schuster (2002). It is well characterized by branching type, large underleaves etc. In the Kerguelen area, only one additional species of Lophozia in the broad sense is known, the until now very imperfectly known species Lophozia leucorhiza from Kerguelen and Heard Is. Lophozia lancistipa and Lophozia leucorhiza are clearly different, not only in the branching type (Lophozia leucorhiza has exclusively terminal branching of Frullania-type, whereas Lophozia lancistipa has lateral-intercalary branching of Andrewsianthus- and Plagiochila-type), but in underleaf form and size, leaf form etc. Schuster (1966) placed Lophozia leucorhiza in the subgenus Orthocaulis (Buch 1933) Schuster (1951) where it seems to be an alien element. Placement in the genus Protolophozia may be better.

Although the generic and familial placement of the other species placed in Protolophozia or Lophozia subgen. Protolophozia is uncertain, they cannot be included in Lophozia s.str. in the current concept. Thus we transfer them to Protolophozia awaiting inclusion in molecular studies. 


\section{Formal treatment}

The format of this note follows what is outlined in Söderström et al. (2012).

Protolophozia androgyna R.M.Schust. ex Váňa et L.Söderstr., sp. nov.

Based on:-Lophozia androgyna R.M.Schust., Beih. Nova Hedwigia 119: 266, 2002 (Schuster 2002) nom. inval. (Art. 36.1; no Latin description).

Type:-AUSTRALIA. Tasmania: Schuster RMS 50513 (holotype F).

Note:-The description in Schuster (2002: 266, note 7) may serve as a valid diagnosis of the taxon.

Protolophozia autoica (R.M.Schust.) Váňa et L.Söderstr. comb. nov.

Basionym:-Lophozia autoica R.M.Schust., Nova Hedwigia 15: 479, 1968 (Schuster 1968).

Type:-NEW ZEALAND. South I.: Otago, Morrison Creek, Leith Valley, Dunedin, ca. 200-300 ft., Schuster RMS $48593 a$ (holotype F).

Protolophozia crispata (R.M.Schust.) Váňa et L.Söderstr. comb. nov.

Basionym:-Lophozia crispata R.M.Schust., Nova Hedwigia 15: 474, 1968 (Schuster 1968).

Type:-ARGENTINA. Tierra del Fuego: Rio Harubre Valley, S of Paso Garibaldi, NE of Ushuaia, Schuster RMS 59417b (holotype F).

Protolophozia druceae (Grolle et E.A.Hodgs.) Váňa et L.Söderstr. comb. nov.

Basionym:-Lophozia druceae Grolle et E.A.Hodgs., J. Roy. Soc. New Zealand 2: 112, 1972 (Hodgson 1972).

Type:-NEW ZEALAND. North Is.: Mt. Pukeamaru, E. Cape, September 1959, Alison C. Druce (holotype MPN 19436 ex herb. Hodgson herb. no. 11442!).

Protolophozia herzogiana (E.A.Hodgs. et Grolle) Váňa et L.Söderstr. comb. nov.

Basionym:-Lophozia herzogiana E.A.Hodgs. et Grolle, Rev. Bryol. Lichénol. 31:152, 1962 [1963] (Grolle 1963).

Type:-NEW ZEALAND. North Is.: N of Rotorua, near Atiamuri, 1931, Allison (holotype MPN 19439 ex herb. Hodgson no. 11881, isotype CHR ex herb. Allison H2528!).

Protolophozia lancistipa (Grolle) Váňa et L.Söderstr. comb. nov.

Basionym:-Andrewsianthus lancistipus Grolle, Bakker, Winterbottom \& Dyer, Marion Prince Edw. Is: 230, 1971 (Grolle 1971).

Type: MARION I. Swamp 1/2 mile SW of weather station, 20 m, 1971, van Zinderen Bakker (holotype JE!).

$\equiv$ Lophozia lancistipa (Grolle) R.M.Schust., J. Hattori Bot. Lab. 78: 121, 1995 (Schuster 1995).

Protolophozia leucorhiza (Mitt.) Váňa et L.Söderstr. comb. nov.

Basionym:-Jungermannia leucorhiza Mitt., J. Linn. Soc., Bot. 15: 68, 1877 [1876] (Mitten 1876).

Lectotype (here designated):-KERGUELEN Is. Voyage of H. M. S. Challenger, Moseley (NY!, isolectotype BM!, G265079 !).

三Lophozia leucorhiza (Mitt.) R.M.Schust., Rev. Bryol. Lichénol. 34 : 273, 1966 (Schuster 1966).

Protolophozia longiflora (Herzog) L.Söderstr. et Váňa, comb. nov.

Basionym:-Orthocaulis longiflorus Herzog, Rev. Bryol. Lichénol. 23: 32 (Herzog 1954).

Type:-CHILE. Westpatagonien: Pte. Puyuhuapi, Rio Ventisquero, G.H. Schwabe n.22/b (holotype JE!).

Note:-Orthocaulis longiflorus is a long forgotten taxon only mentioned, as Lophozia anomala, and briefly discussed by Schuster (2002). Study of the type shows no major difference from Lophozia (Protolophozia) gymnocoleopsis based on the description in Schuster (2002) except for a larger proportion of 3-lobed leaves than described for the latter. 
Leaves are twice as long as wide, subtransverse and shallowly 2-3-lobed. Lobes obtuse to rounded. Male bracts tristichous. Branching lateral-intercalary. The two shoots with perianths in the type specimen are in very bad shape and almost without any leaves.

$\equiv$ Lophozia anomala R.M.Schust. nom. nov., Rev. Bryol. Lichénol. 34: 274, 1966 (Schuster 1966). Blocking name:-Lophozia longiflora (Nees) Schiffn., Sitzungsber. Deutsch. Naturwiss.-Med. Vereins Böhmen "Lotos" Prag 51: 257, 1903 (Schiffner 1903).

= Lophozia gymnocoleopsis R.M.Schust. et J.J.Engel, J. Bryol. 8: 469, 1975 (Schuster \& Engel 1975), syn. nov. Type:-CHILE, Prov. Magallanes, head of Fiordo Peel, leg. Schuster 69-2457 (holotype F-39366).

Protolophozia monoica (E.A.Hodgs.) Váňa et L.Söderstr. comb. nov.

Basionym:-Metahygrobiella monoica E.A.Hodgs., Trans. \& Proc. Roy. Soc. New Zealand, Bot. 3 : 76,1965 (Hodgson 1965).

Type:-NEW ZEALAND. South Is.: Fiordland, mountains above Nancy Sound, 3600 ft., January 1963, Burrows s.n. (MPN, herb. Hodgson no. 12548).

$\equiv$ Lophozia monoica (E.A.Hodgs.) J.J.Engel, Novon 17:311, 2007 (Engel 2007).

Protolophozia multicuspidata (Hook.f. et Taylor) Váňa et L.Söderstr. comb. nov.

Basionym:-Jungermannia multicuspidata Hook.f. et Taylor, London J. Bot. 3: 375, 1844 (Hooker \& Taylor 1844).

Lectotype (here designated):-CAMPBELL IS. J. D. Hooker s.d., s.n. (FH-herb. Taylor!, isolectotype NY-961528!).

三Lophozia multicuspidata (Hook.f. et Taylor) Grolle, Rev. Bryol. Lichénol. 32:155, 1963 [1964] (Grolle 1964).

Protolophozia nivicola (R.M.Schust.) Váňa et L.Söderstr. comb. nov.

Basionym:-Lophozia nivicola R.M.Schust., Nova Hedwigia 15: 477, 1968 (Schuster 1968).

Type:-NEW ZEALAND. South I.:, Mt. Brewster, NE of Haast R., at 4,700 ft., in tussock zone, Schuster RMS 67-455c (holotype F).

Protolophozia perssoniana (H.A.Mill.) Váňa et L.Söderstr. comb. nov.

Basionym:-Lophozia perssoniana H.A.Mill., Ark. Bot. n.s. 5: 508, 1963 (Miller 1963).

Type:-HAWAII. Maui: Mt. Kukui, 9 October 1922, Skottsberg, n.753 (S-B35657!).

Protolophozia subalpina (R.M.Schust.) Váňa et L.Söderstr. comb. nov.

Basionym:-Lophozia autoica var. subalpina R.M.Schust., Nova Hedwigia 15: 482, 1968.

Type:-NEW ZEALAND. South I.: Peaty soil, on boggy ground, track from Lake MacKenzie to Harris Saddle, ca. 3,800 ft., Humboldt Mts., Fiordland, Schuster RMS 67-3888a (holotype F).

$\equiv$ Lophozia subalpina (R.M.Schust.) R.M.Schust., Beih. Nova Hedwigia 119: 263, 2002 (Schuster 2002).

Protolophozia tasmanica (R.M.Schust.) Váňa et L.Söderstr. comb. nov.

Basionym:-Lophozia tasmanica R.M.Schust., Nova Hedwigia 15: 484, 1968 (Schuster 1968).

Holotype:-AUSTRALIA. Tasmania: Above Camp Creek, Surprise Valley, Lyell Hwy., between Derwent Bridge and Queenstown, ca. 2-3 mi. W. of King William Saddle, ca. 2,000 ft., Schuster RMS 50261 (holotype F).

Protolophozia verruculosa (R.M.Schust.) Váňa et L.Söderstr. comb. nov.

Basionym:-Lophozia verruculosa R.M.Schust., Phytologia 39: 242, 1978 (Schuster 1978).

Type:-VENEZUELA. Estado Tachira: S of Villa Paez, Páramo de Tama, peaty wet ground, $3130 \mathrm{~m}$, lower edge of páramo formation, 1 March 1976, Schuster RMS \& L. Ruiz-Terán 76-1944 (holotype F, isotype MERF!). 


\section{Acknowledgement}

We thank Lucia Kawasaki (F) for helping us with information about types of R.M. Schuster in F.

\section{References}

Buch, H. (1933 “1932”) Vorarbeiten zu einer Lebermoosflora Fenno-scandias. I. Ein Versuch zur Aufspaltung der Gattungen Lophozia Dum. und Sphenolobus Steph. Memoranda societatis pro fauna et flora fennica 8: 282-297.

De Roo, R.T., Hedderson, T.A. \& Söderström, L. (2007) Molecular insights into the phylogeny of the leafy liverwort family Lophoziaceae Cavers. Taxon 56: 301-314.

Dumortier, B.C. (1831) Sylloge Jungermannidearum Europae indigenarum, earum genera et species systematice complectens. J. Casterman, Tournay, 100 pp. http://dx.doi.org/10.5962/bhl.title.22343

Dumortier, B.C. (1835) Recueil d'Observations sur les Jungermanniacées. I. Révision des genres. J.-A. Blanquart, Tournay, $27 \mathrm{pp}$.

Engel, J.J. (2007) Studies of New Zealand Hepaticae. 20-38. A miscellanea of new taxa and combinations. Novon 17: 310-314. http://dx.doi.org/10.3417/1055-3177(2007)17[310:SONZHA]2.0.CO;2

Engel, J.J. \& Glenny, D. (2008) A flora of the liverworts and hornworts of New Zealand, Volume 1. Missouri Botanical Garden, St. Louis.

Grolle, R. (1963 “1962”) Eine bemerkenswerte neue Lophozia aus Neuseeland. Revue Bryologique et Lichénologique 31: $152-156$

Grolle, R. (1964 “1963”) Über Kurzia v. Martens. Revue Bryologique et Lichénologique 32: 166-180.

Grolle, R. (1971) Hepaticopsida. In: van Zinderen Bakker, E.M., Winterbottom, J.M. \& Dyer, R.A. (Eds.), Marion and Prince Edward Islands. Report on the South African Biological and Geological Expedition 1965-1966. A. A. Balkema, Cape Town, pp. 228-236.

Herzog, T. (1954) Zur Bryophytenflora Chiles. Revue Bryologique et Lichénologique 23: 27-99.

Hodgson, E.A. (1965) New Zealand Hepaticae (Liverworts) - XVI A miscellany of new genera, new species and notes, part I. Transactions of the Royal Society of New Zealand, Botany 3: 67-97.

Hodgson, E.A. (1972) New Zealand Hepaticae (Liverworts) - XX: A miscellany taxonomic notes, Part 3. Journal of the Royal Society of New Zealand 2: 109-118. http://dx.doi.org/10.1080/03036758.1972.10423309

Hooker, J.D. \& Taylor, T. (1844) Hepaticae Antarcticae; being characters and brief descriptions of the Hepaticae discovered in the southern circumpolar regions during the voyage of H.M. Discovery ships Erebus and Terror. I. species of Lord Auckland's and Campbell's Islands. London Journal of Botany 3: 366-400.

Hübener, J.W.P. (1834) Hepaticologia Germanica oder Beschreibung der deutschen Lebermoose. Schwann and Götz, Mannheim, 314 pp.

Konstantinova, N.A. \& Czernjadieva, I.V. (1995) Petjenotjniki srednego tetjenija r. Sob' (Poljarnyj Ural) [De hepaticis in regione fluxus medii fl. Sob (Ural polaris) obviis notula]. Novosti sistematiki nizshikh rastenij 30: 110-121.

Miller, H.A. (1963) Notes on Hawaiian Hepaticae. V. Collections from recent Swedish expeditions. Arkiv för Botanik, n.s. 5: 489-531.

Mitten, W. (1876) The Musci and Hepaticae collected by H. N. Moseley, M. A., naturalist to H. M. S. 'Challenger'. Journal of the Linnean Society. Botany 15 “1877”: 59-73.

Schiffner, V.F. (1903) Kritische Bemerkungen über die europäischen Lebermoose mit Bezug auf die Exemplare des Exsiccatenwerkes, Hepaticae Europaeae exsiccatae 3 (101-150). Sitzungsberichte des Deutschen Naturwissenschaftlich-Medicinischen Vereins für Böhmen "Lotos" in Prag 51: 213-275.

Schljakov, R.N. (1979) Novye dopolneniya k flore pethenochnikov severnykh rayonov SSSR [Addidamenta nova as floram hepaticarum regionum septentrionalium URSS]. Novosti sistematiki nizshikh rastenij 16: 201-208.

Schuster, R.M. (1951 “1950”) Notes on nearctic Hepaticae. II. The Hepaticae in east coast of Hudson Bay. Bulletin of the National Museum of Canada 122: 1-62.

Schuster, R.M. (1966) Studies in Lophoziaceae. 1. The genera Anastrophyllum and Sphenolobus and their segregates. 2. Cephalolobus gen. n., Acrolophozia gen. n. and Protomarsupella gen. n. Revue Bryologique et Lichénologique 34: 241-287.

Schuster, R.M. (1968) Studies on the Hepaticae, XXIX-XLIV. A miscellany of new taxa and new range extensions. Nova Hedwigia 15: 437-529.

Schuster, R.M. (1969) The Hepaticae and Anthocerotae of North America. vol. II. Columbia University Press, New York, $1062 \mathrm{pp}$.

Schuster, R.M. (1978) Studies on Venezuelan Hepaticae. I. Phytologia 39: 239-251.

Schuster, R.M. (1995) The hepaticae of the Prince Edward Islands. II. On Gymnocoleopsis (Schust.) Schust., Lophozia 
cylindriformis (Mitt.) Steph. and the subgeneric classification of the genus Lophozia Dumort. Journal of the Hattori Botanical Laboratory 78: 119-135.

Schuster, R.M. (2002) Austral Hepaticae, part II. Beihefte zur Nova Hedwigia 119: 1-606.

Schuster, R.M. \& Damsholt, K. (1987) Some new taxa of Jungermanniales. Phytologia 63: 325-328.

Schuster, R.M. \& Engel, J.J. (1975) Austral Hepaticae, IV. Notes on Lophozia subgenus Protolophozia Schust., with diagnosis of a new South American species. Journal of Bryology 8: 465-474.

Söderström, L. Hagborg, A. \& von Konrat, M. (2012) Notes on Early Land Plants Today. Phytotaxa 65: 41-42.

Söderström, L., Weibull, H. \& Damsholt, K. (2000) A new species of Lophozia (subgen. Protolophozia) from Fennoscandia. Lindbergia 25: 3-7.

Söderström, L., De Roo, R. \& Hedderson, T. (2010) Taxonomic novelties resulting from recent reclassification of the Lophoziaceae/Scapaniaceae clade. Phytotaxa 3: 47-53.

Stephani, F. (1902) Species Hepaticarum 2. Bulletin de l'Herbier Boissier, série 2 2: 35-48.

Vilnet, A.A., Konstantinova, N.A. \& Troitsky, A.V. (2010) Molecular insight on phylogeny and systematics of the Lophoziaceae, Scapaniaceae, Gymnomitriaceae and Jungermanniaceae. Arctoa 19: 31-50.

Vilnet, A.A., Konstantinova, N.A. \& Troitsky, A.V. (2011) Taxonomical rearrangements of Solenostomataceae (Marchantiophyta) with description of a new family Endogemmataceae based on trnL-F cpDNA analysis. Folia Cryptogamica Estonica 48: 125-133. 